\title{
Long-Term Course to Lumbar Disc Resorption Patients and Predictive Factors Associated with Disc Resorption
}

\author{
Jinho Lee, Joowon Kim, Joon-Shik Shin, Yoon Jae Lee, Me-riong Kim, \\ Seon-Yeong Jeong, Young-jun Choi, Tae Kyung Yoon, Byung-heon Moon, Su-bin Yoo, \\ Jungsoo Hong, and In-Hyuk Ha
}

Jaseng Spine and Joint Research Institute, Jaseng Medical Foundation, Seoul, Republic of Korea

Correspondence should be addressed to In-Hyuk Ha; hanihata@gmail.com

Received 18 April 2017; Accepted 5 June 2017; Published 9 July 2017

Academic Editor: Gihyun Lee

Copyright (C) 2017 Jinho Lee et al. This is an open access article distributed under the Creative Commons Attribution License, which permits unrestricted use, distribution, and reproduction in any medium, provided the original work is properly cited.

\begin{abstract}
The long-term course to lumbar intervertebral disc herniation (LDH) patients receiving integrative Korean medicine treatment and predictive factors associated with disc resorption were investigated. $\mathrm{LDH}$ patients who received integrative Korean medicine treatment from February 2012 to December 2015 and were registered in the "longitudinal project for LDH on MRI" were included. Disc resorption amount was measured 3-dimensionally with disc degeneration and modic change levels on baseline and follow-up MRIs. Patient characteristics, Korean medicine use, pain, symptom recurrence, and satisfaction were assessed through medical records and phone surveys. Of 505 participants, 19 did not show disc resorption, while 486 did. A total of 220 displayed resorption rates of $\geq 50 \%$. LDH volume at baseline was $1399.82 \pm 594.96 \mathrm{~mm}^{3}$, and that on follow-up MRI was $734.37 \pm 303.33 \mathrm{~mm}^{3}$, indicating a $47.5 \%$ decrease $(p<0.0001)$. Predictive factors for disc resorption were disc extrusion, Komori migration classification, and LDH amount. Approximately $68.4 \%$ did not experience symptom recurrence over the $51.86 \pm 19.07$-month follow-up, and $90.3 \%$ were satisfied with Korean medicine treatment. The majority of LDH patients who improved after integrative Korean medicine treatment showed disc resorption within 1 year with favorable long-term outcomes. Predictive factors for disc resorption should be duly considered for informed decision-making. This trial is registered with ClinicalTrials.gov NCT02841163.
\end{abstract}

\section{Introduction}

Recent developments in the modernization of traditional Oriental medicine include greater integration with medical technology and devices toward greater efficacy, safety, and diagnostic and prognostic accuracy. A dual medical system of conventional medicine and traditional Korean medicine is employed in Korea, and conventional diagnostic imaging such as X-rays and magnetic resonance imaging (MRI) and advances in technology may be used to further promote the modernization of traditional Oriental medicine by means of an integrative and collaborative approach.

Prevalence estimates for sciatica have been reported to range from $1.6 \%$ in the general population to $43 \%$ in select working populations, and the most commonly cited cause of sciatica is lumbar intervertebral disc herniation (IDH) [1]. Low back pain (LBP) and sciatica incur most time off work and disability of all medical conditions [2], and LBP from spinal diseases such as spondylosis and intervertebral disc disorders were the 2nd highest ranking reason for hospitalized care in the working population (age 18-44) in the US in 2005 [3]. Approximately 317,000 lumbar surgeries were performed in the US in 1997 [4], which has steadily risen to 1 million spinal procedures in 2002 [5].

However, the natural history of lumbar IDH is favorable, and $70 \%$ of patients recover from sciatica without surgery within 6 weeks [6]. The 10 -year observation results of surgical and nonsurgical treatment show no significant difference after 4 years [7], and a recent large-scale study on acute IDH reported that while early surgical intervention may be more beneficial economically through swift recovery and return to work, 1-year pain and function results did not differ significantly from nonsurgical treatment [8]. An estimated $10 \%$ of lumbar IDH patients consider surgery due 
to continuous pain and/or neurological deficit, and accurate prediction of development into chronic pain is therefore of importance [6]. Advances in radiological examination have revealed how IDH may be spontaneously resorbed in time with resolution of neurological symptoms [9], and various factors predictive of disc resorption are being studied as they hold valuable information on chronicization and need for surgical intervention [10-16].

In Korea, many IDH patients opt for primary care at Korean medicine institutions to receive Korean medicine treatment and avoid surgery. The authors have previously reported the long-term outcomes and risk factors for poor prognosis in lumbar IDH patients receiving integrative Korean medicine treatment, excluding conventional treatment $[17,18]$. The objectives of this study were to observe the long-term course to IDH patients with spontaneous disc resorption after integrative Korean medicine treatment and identify predictive factors associated with resorption amount.

\section{Materials and Methods}

Jaseng Hospital of Korean Medicine, certified by the Korean Ministry of Health and Welfare as a spine-specialty Korean medicine hospital, operates 19 branches which treat 900,000 spinal disorder patients per year [19]. Electronic medical records (EMRs) and radiological assessments of participants who were diagnosed with lumbar IDH on MRI, received integrative Korean medicine treatment at these medical institutions from February 2012 to December 2015, and were included in the "longitudinal project for IDH on MRI" were assessed. The aim of the project was to assess differences in diagnostic imaging before and after treatment by allowing physicians to select 1-2 patients annually for complimentary follow-up MRIs with written consent for use of data for academic means. Survey assessments were conducted by phone from April to October 2016. This study received approval from the Institutional Review Board of Jaseng Hospital of Korean Medicine (JASENG 2016-06-003).

2.1. Participants. The inclusion criteria were as follows: (1) patients diagnosed with lumbar IDH on MRI, (2) patients who experienced improvement of LBP and/or sciatica from integrative Korean medicine treatment, and (3) written consent to participate in the "longitudinal project for IDH on MRI" and a follow-up MRI. The exclusion criteria were (1) patients who received lumbar spinal surgery during integrative Korean medicine treatment, (2) patients with main complaint of cervical IDH, and (3) patients with MRI errors or low resolution.

2.2. EMR Assessment and Phone Survey. EMRs were reviewed to assess participant characteristics including sex, age, LBP and/or sciatica, and details of integrative Korean medicine treatment (whether the patient underwent hospitalization, days in inpatient and ambulatory care, and frequency of treatment). Phone surveys were also conducted to investigate initial and current levels of pain associated with lumbar IDH as assessed by the numeric rating scale (NRS), whether the patient received recommendation for surgery, experienced recurrence of symptoms, or underwent lumbar surgery, awareness of spontaneous disc resorption, and satisfaction with Korean medicine treatment.

2.3. Analysis of Imaging Results. This study used threedimensional measurements on MRI to determine IDH amount according to previous measurement methods [10]. The authors selected a main IDH level on sagittal sections of T2-weighted MRIs best correlating with symptoms. A line connecting 2 points indicating the posterior edges of the superior and inferior endplates was drawn as reference. The IDH area and amount outside the reference line were demarcated and measured using the "measure area freehand" function in the picture archiving and communication system (PACS) on sagittal sections. The volume was calculated by multiplying the IDH area on each sagittal section with the MRI scan thickness plus the interslice intervals.

The IDH level was classified into bulging, protrusion, extrusion, migration, and sequestration and by the migration criteria suggested by Komori et al. [20] where migrating disc amount is categorized in comparison to the posterior height of the adjacent vertebrae. IDH $<1 / 3$ of the posterior height of the vertebrae immediately superior or inferior to the IDH level was designated as Grade 2, that between $1 / 3$ and $2 / 3$ height as Grade 3, >2/3 height as Grade 4, and no IDH as Grade 1. The IDH degeneration categorization employed the classification criteria put forth by Pfirrmann et al., which classifies degeneration by structure, signal intensity, and disc height on MRI into 5 levels from Grades I to V. Higher grades imply greater degeneration, and generally Grades IV and V are taken to indicate disc degeneration [21]. Modic types were classified into 0 (no modic change), and modic types 1, 2, and 3. The location of modic type change in the vertebral body was recorded as (i) above, (ii) below, or (iii) both above and below the IDH level, or (iv) no modic type change. Two Korean medicine doctors (KMDs) experienced in spinal imaging received training for standardized reading for higher reliability. Interrater reliability was assessed through measurement and classification of 20 random pairs of imaging results (total of $40 \mathrm{MRIs}$ before and after treatment) (see Supplementary Table 1 in Supplementary Material available online at https://doi.org/10.1155/2017/2147408).

2.4. Statistical Analysis. All continuous variables are expressed as mean $\pm \mathrm{SD}$, and categorical variables as $n(\%)$. Consistency of IDH imaging measurement was determined through intraclass correlation coefficient (ICC), and that of IDH and degeneration classification was analyzed by Cohen's kappa. Predictive factors for $\geq 50 \%$ IDH regression on followup MRI were determined through logistic regression analysis considering for major influence variables (age, gender, level of disc degeneration, IDH type, Komori migration classification, modic type, modic change area, time interval between baseline and follow-up MRIs, and total treatment duration). Univariate analysis was performed, and factors that were significant in univariate analysis were included in additional multivariate analyses with age and baseline IDH amount, adjusted for. Comparison of IDH amounts before and after treatment was analyzed using paired $t$-test and IDH type 


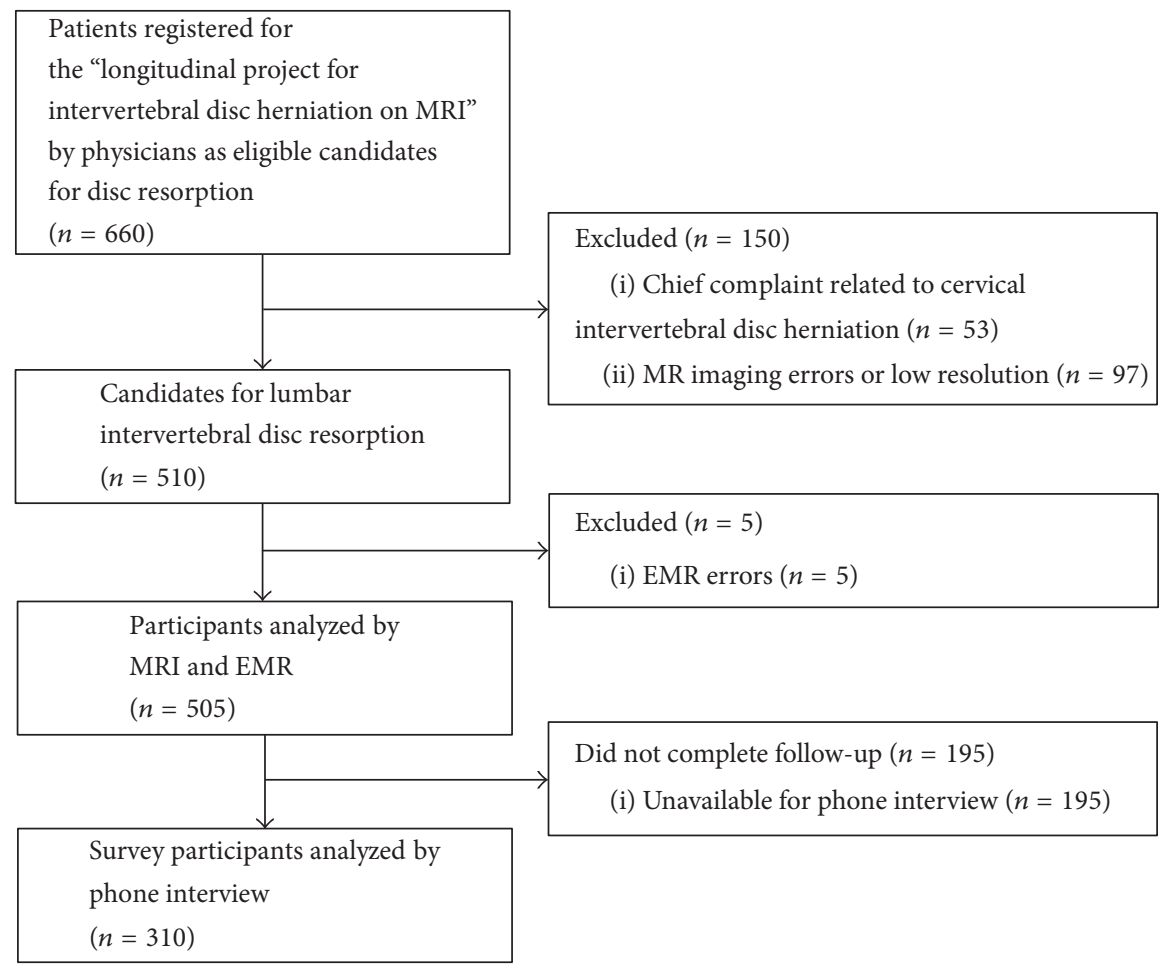

FIGURE 1: Flow diagram of the study.

and level of degeneration were analyzed with chi-square test. Associations between IDH volume on baseline and followup MRIs, change in volume, resorption rate, and age were assessed through regression analysis. All analyses comparing measurements before and after treatment were performed using STATA 14.0 (StataCorp, College Station, Texas, USA).

\section{Results}

A total of 660 patients were registered in the "longitudinal project for IDH on MRI" and underwent baseline and followup MRIs. Of these eligible participants, patients with chief complaint diagnosis of cervical IDH, imaging or EMR errors, and low imaging resolution were excluded, and 505 lumbar IDH patients participated. Phone surveys were performed in all participants except those that the researchers were unable to reach, yielding a total of 310 survey participants (61\%) (Figure 1).

Baseline demographic characteristics of the 505 participants were as follows: Average age was $39.08 \pm 10.19$ years, with higher male percentage (60.6\%), and the most common IDH level was L4/5 (53.3\%) and L5/S1 (38.8\%). The majority did not have LBP $(90.1 \%)$, and most presented with sciatica $(83.6 \%)$ which was mainly unilateral (76.0\%) (Table 1$)$.

The average time interval between baseline and followup MRI was $341.38 \pm 306.83$ days, and the difference between MRIs was analyzed. Evidence of disc resorption was not seen in 19 patients and seen in 486 of whom 220 exhibited a resorption rate of $\geq 50 \%$. The IDH volume at baseline was $1399.82 \pm 594.96 \mathrm{~mm}^{3}$, and that at follow-up was $734.37 \pm 303.33 \mathrm{~mm}^{3}$, indicating a statistically significant volume decrease of $47.5 \%$ ( $p<0.0001)$. In Komori migration classification, $38.6 \%$ of patients were classified as Grade 2 or 3 at baseline, which decreased to $3.4 \%$ at follow-up. Regarding IDH type, the percentage of patients with extrusion or greater herniation level (i.e., extrusion, migration, or sequestration) at baseline was $87.9 \%$, which declined to $22.8 \%$ at followup. Disc degeneration and modic type grade did not show significant difference (Table 2).

Of the 505 participants, 186 (38.6\%) were hospitalized while the others received integrative Korean medicine treatment in the outpatient department. The number of days in inpatient care was $34.34 \pm 29.53$ days, that of outpatient visits was $32.88 \pm 20.72$ days, and the total number of days in care was $45.53 \pm 28.61$ days. The vast majority of patients were given herbal medicine (96.4\%), acupuncture (96.4\%), and Chuna manipulation $(87.9 \%)$, followed by bee venom pharmacopuncture $(65.0 \%)$, pharmacopuncture $(53.3 \%)$, electroacupuncture (46.5\%), and cupping (22.6\%). Of herbal medicine and pharmacopuncture, Chungpa-jun and variations, of which GCSB-5 is the main ingredient [22-25], and Shinbaro pharmacopuncture [26] were used most frequently, respectively (Table 3).

The mean time interval between baseline and follow-up phone interviews in the 303 survey participants was $51.86 \pm$ 19.07 months. Baseline pain NRS was reported at $8.34 \pm 1.42$, which was put at $1.27 \pm 1.57$ for current pain. Lumbar surgery had previously been recommended for around half of all patients at baseline (54.2\%), and $73.2 \%$ were not aware of spontaneous disc resorption. Ninety-four patients reported pain recurrence(s) during the follow-up period (30.3\%), and the majority chose Korean medicine for its treatment. 
Most patients were satisfied with Korean medicine (90.1\%) and nonsurgical methods for lumbar IDH treatment were recommended for them $(94.2 \%)$ (Table 4$)$.

Analysis of predictive factors for $\geq 50 \%$ resorption (dichotomous variable) in IDH resorption amount revealed that IDH type and amount (Komori migration classification) were significantly associated. When Komori migration classification Grade 1 was set as reference, the odds ratios (ORs) for Grades 2 and 3 were 1.7 (95\% CI 1.04-2.76) and 2.46 (95\% CI 1.37-4.42), respectively. When protrusion was designated as the reference in IDH type, the ORs for extrusion and migration were 2.49 (95\% CI 1.10-5.60) and 6.3 (95\% CI 2.58-15.42), respectively (Table 5). Baseline IDH amount was shown to be positively related to disc resorption rate (continuous variable), whereas age was negatively associated (Figure 2).

\section{Discussion}

Of 505 participants who were mainly middle-aged males presenting with unilateral sciatica symptoms due to IDH at L4/5 and L5/S1 levels, 486 displayed spontaneous disc resorption $(96.2 \%)$, and 220 showed resorption rates of $\geq 50 \%$ (43.6\%). In Komori migration classification, $38.6 \%$ of patients were classified as Grades 2 or 3 at baseline which decreased to $3.4 \%$ at follow-up, and $87.9 \%$ were classified as disc extrusion or higher herniation level on baseline MRI, compared to $22.8 \%$ at follow-up regarding IDH type. Major factors determined to predict disc resorption were baseline IDH type, Komori migration classification, and age. Over a $51.86 \pm 19.07$-month follow-up period, $68.4 \%$ did not experience LBP recurrence, and $90.3 \%$ replied that they were satisfied with Korean medicine treatment.

The underlying mechanism of disc resorption has been suggested to be enzymatic degradation and phagocytosis of IDH matter through inflammatory reaction and vascularization. As IDH material enters the vascularized epidural space, it is identified by the body as a foreign substance, leading to immune and inflammatory response, and thus induces neovascularization, enzymatic degradation, and macrophage phagocytosis. This consequently leads to production of matrix proteinases and increased cytokine levels, resulting in disc resorption $[27,28]$.

The results of this study are consistent with other studies where sequestrated disc matter was shown to be better resorbed than protruded disc. This is speculated to be due to the degree of penetration (tear) of the annulus fibrosus and posterior longitudinal ligament and increased exposure to systemic circulation within the epidural space $[29,30]$.

A recent systematic review on the probability of spontaneous disc resorption by IDH type including 9 papers covering 361 patients reported that $96 \%$ of disc sequestration (resorption in 52/54 patients), $70 \%$ of disc extrusion (108/154), $41 \%$ of disc protrusion (38/93), and $13 \%$ of disc bulging types (8/60) were resorbed, indicating high resorption rates in sequestration and extrusion types [12]. Similarly, Komori et al. reported that although $78 \%$ of migrated discs resulted in resorption (resorption in 36/64 patients), only $17 \%(7 / 48)$ were resorbed in nonmigrated discs [20], and
TABLE 1: Baseline demographic characteristics of participants of the "longitudinal project for IDH on MRI".

\begin{tabular}{|c|c|c|c|}
\hline \multirow{2}{*}{ Variables } & \multicolumn{3}{|c|}{$n=505$} \\
\hline & $n$ & $\%$ & Mean (SD) \\
\hline Age (years) & & & $39.08 \pm 10.19$ \\
\hline$<25$ & 23 & 4.6 & \\
\hline$\geq 25-<55$ & 436 & 86.3 & \\
\hline$\geq 55$ & 46 & 9.1 & \\
\hline \multicolumn{4}{|l|}{ Sex } \\
\hline Male & 306 & 60.6 & \\
\hline Female & 199 & 39.4 & \\
\hline \multicolumn{4}{|l|}{ Herniated disc level } \\
\hline $\mathrm{L} 1 / 2$ & 2 & 0.4 & \\
\hline $\mathrm{L} 2 / 3$ & 9 & 1.8 & \\
\hline $\mathrm{L} 3 / 4$ & 29 & 5.7 & \\
\hline $\mathrm{L} 4 / 5$ & 269 & 53.3 & \\
\hline $\mathrm{L} 5 / \mathrm{S} 1$ & 196 & 38.8 & \\
\hline \multicolumn{4}{|l|}{ Low back pain } \\
\hline No & 454 & 90.1 & \\
\hline Yes & 48 & 9.5 & \\
\hline \multicolumn{4}{|l|}{ Radiating leg pain } \\
\hline No & 80 & 15.8 & \\
\hline Yes, radiating to thigh & 127 & 25.2 & \\
\hline Yes, radiating to calf & 239 & 47.3 & \\
\hline Yes, but with no indication of area & 56 & 11.1 & \\
\hline Unknown & 3 & 0.6 & \\
\hline \multicolumn{4}{|l|}{ Bilateral radiating leg pain } \\
\hline No & 432 & 85.5 & \\
\hline Yes & 66 & 13.1 & \\
\hline Unknown & 7 & 1.4 & \\
\hline \multicolumn{4}{|l|}{ Unilateral radiating leg pain } \\
\hline No & 115 & 22.8 & \\
\hline Yes & 384 & 76.0 & \\
\hline Unknown & 6 & 1.2 & \\
\hline
\end{tabular}

IDH, intervertebral disc herniation; MRI, magnetic resonance imaging; SD, standard deviation.

went on to publish that whereas the complete resolution rate of migrated discs was $41 \%$, that of nonmigrated discs was $0 \%$ [31]. In a study by Ahn et al., 25 out of 36 discs showed reduction in size, where $56 \%$ of subligamentous, $79 \%$ of transligamentous, and $100 \%$ of sequestered disc herniation resulted in IDH size reduction, and the study concluded that transligamentous extension and posterior longitudinal ligament rupture were the most influential factors in IDH reduction [29]. Moreover, in a 2014 observational study comparable to the current study on 102 patients receiving traditional Chinese medicine treatment, $81.37 \%$ reported symptom improvement and $18.63 \%$ were considered to require surgery. IDH volume decreased from $1433.89 \pm 525.49 \mathrm{~mm}^{3}$ to $1002.01 \pm$ $593.95 \mathrm{~mm}^{3}$, resulting in an average resorption rate of $27.25 \pm$ $32.97 \%$, and 20 patients presented resorption rates of $\geq 50 \%$ [10]. While modic change was not recognized as a predictive factor of resorption in this study, a 2014 study by Shan et al. 
TABLE 2: Baseline and follow-up MRI results.

\begin{tabular}{|c|c|c|c|c|c|}
\hline \multirow{2}{*}{ Variables } & \multicolumn{2}{|c|}{ Baseline MRI } & \multicolumn{2}{|c|}{ Follow-up MRI } & \multirow{2}{*}{$p$ valu } \\
\hline & $n$ & $\%$ & $n$ & $\%$ & \\
\hline Volume of herniated disc $\left(\mathrm{mm}^{3}\right)^{\mathrm{a}}$ & $1399.82 \pm 594.96$ & & $734.37 \pm 303.33$ & & 0.000 \\
\hline Time interval between baseline and follow-up MRIs (days) & $341.38 \pm 306.83$ & & & & \\
\hline \multicolumn{6}{|l|}{ Resorption rate (\%) } \\
\hline Aggravation & 19 & 3.76 & & & \\
\hline$>0-\leq 25$ & 75 & 14.85 & & & \\
\hline$>25-\leq 50$ & 191 & 37.82 & & & \\
\hline$>50-\leq 75$ & 205 & 40.59 & & & \\
\hline$>75-\leq 100$ & 15 & 2.97 & & & \\
\hline \multicolumn{6}{|l|}{ Komori migration classification $^{\mathrm{b}}$} \\
\hline 1 & 310 & 61.4 & 488 & 96.6 & \\
\hline 2 & 106 & 21.0 & 16 & 3.2 & 0.000 \\
\hline 3 & 89 & 17.6 & 1 & 0.2 & \\
\hline \multicolumn{6}{|l|}{ Disc herniation type ${ }^{b}$} \\
\hline Bulging & 1 & 0.2 & 87 & 17.2 & \\
\hline Protrusion & 60 & 11.9 & 303 & 60.0 & \\
\hline Extrusion & 328 & 65.0 & 100 & 19.8 & 0.000 \\
\hline Migration & 93 & 18.4 & 12 & 2.4 & \\
\hline Sequestration & 23 & 4.6 & 3 & 0.6 & \\
\hline \multicolumn{6}{|l|}{ Disc degeneration grade ${ }^{b}$} \\
\hline $\mathrm{I}$ & 6 & 1.2 & 4 & 0.8 & \\
\hline II & 47 & 9.3 & 39 & 7.7 & \\
\hline III & 215 & 42.6 & 202 & 40.0 & 0.582 \\
\hline IV & 224 & 44.4 & 243 & 48.1 & \\
\hline $\mathrm{V}$ & 13 & 2.6 & 17 & 3.4 & \\
\hline \multicolumn{6}{|l|}{ Modic change type of vertebral body ${ }^{\mathrm{b}}$} \\
\hline 0 & 358 & 70.9 & 353 & 69.9 & \\
\hline 1 & 16 & 3.2 & 14 & 2.8 & 0.930 \\
\hline 2 & 128 & 25.3 & 134 & 26.5 & \\
\hline 3 & 3 & 0.6 & 4 & 0.8 & \\
\hline \multicolumn{6}{|l|}{ Modic change of vertebral body area ${ }^{b}$} \\
\hline Above the herniated disc & 22 & 4.4 & 20 & 4.0 & \\
\hline Below the herniated disc & 20 & 4.0 & 20 & 4.0 & 0.981 \\
\hline Both above and below the herniated disc & 105 & 20.8 & 109 & 21.6 & \\
\hline None & 358 & 70.9 & 356 & 70.5 & \\
\hline
\end{tabular}

${ }^{a}$ Paired $t$-test was used in analysis of change in amount of herniated disc matter from baseline; ${ }^{b}$ Chi-square test was used in analysis of change in classification of herniated disc from baseline; MRI, magnetic resonance imaging.

reported that whereas 35 of 85 patients in the modic change group (of whom the majority were of Type 2) did not show much difference in IDH size, the group with no modic change displayed significant decrease [32]. Iwabuchi et al. purported that resorption factors could be identified on T1- and T2weighted MRIs, with high signal intensity of IDH areas in T1and T2-weighted MRIs in the nonregression group [33].

The largest strength of this study is that, to the best of our knowledge, it covers the largest sample size $(n=$ 505) of studies addressing disc resorption. This is especially important in research on predictive factors for disc resorption as it is difficult for small sample sized studies to secure sufficient statistical power. Furthermore, while predictive factor studies should consider various factors through prediction models using multivariate analysis, only univariate analysis may be possible in studies with small sample size. Another major strength of this study is that IDH was measured 3-dimensionally on MRI. Two-dimensional crosssectional images may differ greatly from positioning, and various limitations and errors arise in measurement from the sectional directions in image acquisition. This study also includes long-term observation results at an average of 51.86 
TABLE 3: Use of integrative Korean medicine treatment.

\begin{tabular}{lccc}
\hline Variables & & & $n=505$ \\
Days (mean \pm SD) \\
\hline Hospitalized care & 186 & 36.8 & $34.34 \pm 29.53$ \\
Number of hospitalized days & & & $32.88 \pm 20.72$ \\
Number of outpatient visit days & & & $45.53 \pm 28.61$ \\
Total number of treatment days & & & $136.35 \pm 90.81$ \\
Frequency of integrative Korean medicine treatment & & 96.4 & $31.15 \pm 28.62$ \\
$\quad$ Herbal medicine & 487 & 53.3 & $26.78 \pm 20.38$ \\
Pharmacopuncture & 269 & 65.0 & $39.06 \pm 35.08$ \\
Bee venom pharmacopuncture & 328 & 87.9 & $45.42 \pm 32.79$ \\
Chuna manipulation & 444 & 96.4 & $32.80 \pm 22.42$ \\
Acupuncture & 487 & 46.5 & $20.00 \pm 17.81$ \\
Electroacupuncture & 235 & 22.6 & \\
Cupping & 114 & & \\
\hline
\end{tabular}

SD, standard deviation.

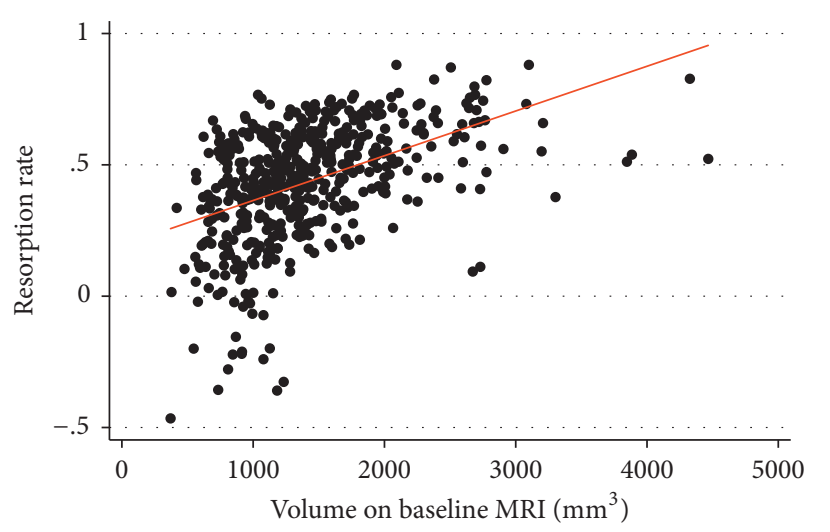

Volume on baseline MRI $\left(\mathrm{mm}^{3}\right)$

Fitted values

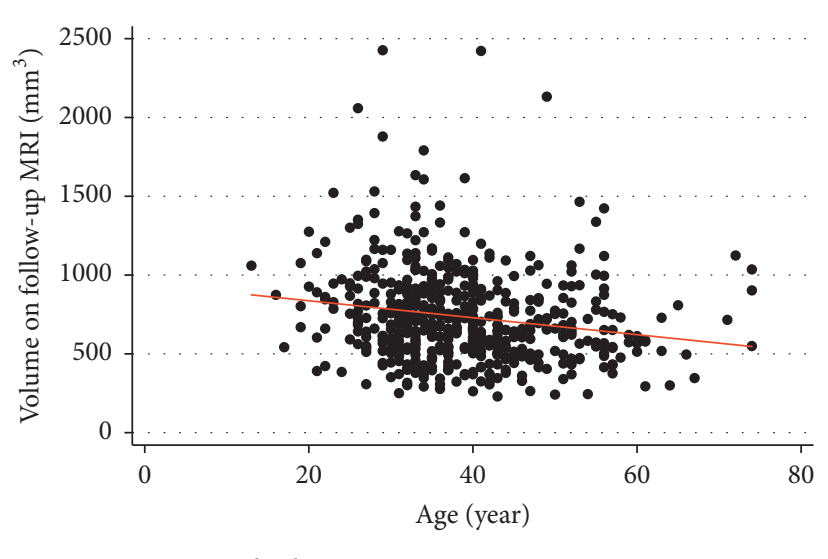

Fitted values

(a)

(b)

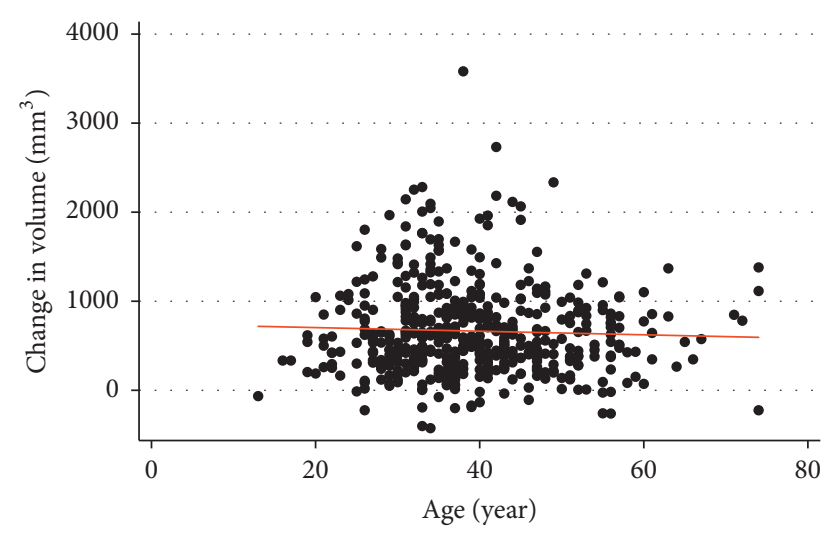

(c)

FIGURE 2: Associations between herniated disc volume on baseline MRI, volume on follow-up MRI, change in volume, disc herniation resorption rate, and age. (a) Association between herniated disc volume on baseline MRI and resorption rate. $y=0.19+0.0001703 x$, $R^{2}=0.2148, p \leq 0.001$. (b) Association between herniated disc volume on follow-up MRI and age. $y=944.39-5.37 x, R^{2}=0.0326$, $p \leq 0.001$. (c) Association between change in herniated disc volume and age. $y=744.62-2.03 x, R^{2}=0.0016, p=0.362$. 
TABLE 4: Outcome measures assessed through phone interview.

\begin{tabular}{|c|c|c|c|}
\hline \multirow{2}{*}{ Variables } & \multicolumn{3}{|c|}{$n=310$} \\
\hline & $n$ & $\%$ & Mean \pm SD \\
\hline Time interval between initial visit and phone interview (months) & & & $51.86 \pm 19.07$ \\
\hline NRS of pain at baseline $\mathrm{a}^{\mathrm{a}}$ & & & $8.34 \pm 1.42$ \\
\hline NRS of current pain & & & $1.27 \pm 1.57$ \\
\hline \multicolumn{4}{|l|}{ Recommendation of surgery ${ }^{\mathrm{a}}$} \\
\hline Recommended for surgery & 168 & 54.2 & \\
\hline Not recommended for surgery & 112 & 36.1 & \\
\hline Do not know & 6 & 1.9 & \\
\hline Did not visit conventional medicine institution & 24 & 7.7 & \\
\hline \multicolumn{4}{|l|}{ Awareness of possibility of disc resorption ${ }^{\mathrm{a}}$} \\
\hline Aware & 83 & 26.8 & \\
\hline Unaware & 227 & 73.2 & \\
\hline \multicolumn{4}{|l|}{ Experience of symptom recurrence ${ }^{a}$} \\
\hline Yes & 94 & 30.3 & \\
\hline No & 212 & 68.4 & \\
\hline Do not know & 4 & 1.3 & \\
\hline \multicolumn{4}{|l|}{ Treatment type used for recurrence ${ }^{a}$} \\
\hline Korean medicine treatment & 104 & 36.1 & \\
\hline Conventional nonsurgical treatment & 14 & 4.9 & \\
\hline Surgery & 7 & 2.4 & \\
\hline No recurrence requiring treatment & 163 & 56.6 & \\
\hline \multicolumn{4}{|l|}{ Satisfaction with Korean medicine treatment } \\
\hline Very satisfied & 159 & 51.5 & \\
\hline Satisfied & 120 & 38.8 & \\
\hline Slightly satisfied & 28 & 9.1 & \\
\hline Unsatisfied & 1 & 0.3 & \\
\hline Very unsatisfied & 1 & 0.3 & \\
\hline \multicolumn{4}{|l|}{ Recommendation of treatment to others } \\
\hline Surgical treatment & 4 & 1.3 & \\
\hline Nonsurgical treatment & 292 & 94.2 & \\
\hline Do not know & 14 & 4.5 & \\
\hline \multicolumn{4}{|l|}{ Effective Korean medicine treatment type } \\
\hline Herbal medicine & 64 & 20.7 & \\
\hline Bee venom pharmacopuncture, pharmacopuncture & 135 & 43.6 & \\
\hline Acupuncture & 26 & 8.4 & \\
\hline Chuna manipulation & 60 & 19.4 & \\
\hline Do not know & 61 & 19.7 & \\
\hline
\end{tabular}

${ }^{a}$ Baseline timepoint was assessed retrospectively at the time of follow-up survey; SD, standard deviation; NRS, numeric rating scale.

months (approximately 4.3 years) in addition to imaging analyses to better portray current state, symptom recurrence, and satisfaction with treatment.

Despite these strengths, this study also suffers from the following weaknesses. The largest limitation is probably the fact that although the "longitudinal project for IDH on MRI" itself was conducted prospectively, outcome measures were partly investigated in a retrospective manner through EMR examination and include limited clinical information. Moreover, although follow-up MRIs were conducted allowing for sufficient time for potential disc resorption as clinical settings and timeframes permitted, the time intervals are inconsistent as a result. The fact that MRI scans were performed using different imaging apparatus and under different conditions at various in-hospital and external sites in this multicenter study may also be viewed as a limitation.

While the natural course of lumbar IDH is widely considered to be favorable, surgical interventions are still common $[4,5]$. International consensus recommends consideration of surgery if symptoms persist after a certain period of conservative treatment [34]; however, consensus has not been reached regarding its duration $[35,36]$. Although severe IDH potentially incurs neurological disability and higher pain levels, it also entails greater possibility of disc resorption. 
TABLE 5: Assessment of predictive factors at baseline associated with herniated disc resorption in participants.

\begin{tabular}{|c|c|c|c|c|}
\hline & \multicolumn{2}{|c|}{ Univariate } & \multicolumn{2}{|c|}{ Multivariate $^{\mathrm{a}}$} \\
\hline & OR & $95 \% \mathrm{CI}$ & OR & $95 \% \mathrm{CI}$ \\
\hline Age (continuous) & 1.01 & $(0.99,1.03)$ & & \\
\hline Sex, male (ref. female) & 0.97 & $(0.67,1.37)$ & & \\
\hline \multicolumn{5}{|l|}{ Disc degeneration grade (ref. I) } \\
\hline II & 3.39 & $(0.37,31.38)$ & & \\
\hline III & 3.14 & $(0.36,27.38)$ & & \\
\hline IV & 4.57 & $(0.53,39.77)$ & & \\
\hline $\mathrm{V}$ & 16.67 & $(1.36,204.03)$ & & \\
\hline \multicolumn{5}{|l|}{ Disc herniation type (ref. protrusion) } \\
\hline Bulging & - & & & \\
\hline Extrusion & 4.66 & $(2.15,10.13)$ & 2.49 & $(1.10,5.60)$ \\
\hline Migration & 11.82 & $(5.02,27.85)$ & 6.3 & $(2.58,15.42)$ \\
\hline Sequestration & 12.190 & $(3.91,37.95)$ & 3 & $(0.84,10.68)$ \\
\hline \multicolumn{5}{|l|}{ Komori migration classification (ref. 1) } \\
\hline 2 & 2.48 & $(1.58,3.89)$ & 1.7 & $(1.04,2.76)$ \\
\hline 3 & 5.46 & $(3.24,9.18)$ & 2.46 & $(1.37,4.42)$ \\
\hline \multicolumn{5}{|l|}{ Modic change type of vertebral body (ref. 0) } \\
\hline 1 & 0.84 & $(0.30,2.37)$ & & \\
\hline 2 & 4.4 & $(0.94,2.10)$ & & \\
\hline 3 & 0.7 & $(0.06,7.81)$ & & \\
\hline \multicolumn{5}{|l|}{ Modic change of vertebral body area (ref. none) } \\
\hline Above the herniated disc & 0.94 & $(0.37,2.35)$ & & \\
\hline Below the herniated disc & 1.410 & $(0.57,3.46)$ & & \\
\hline Both above and below the herniated disc & 1.38 & $(0.90,2.12)$ & & \\
\hline Time interval between MRIs (continuous) & 1 & $(0.99,1.00)$ & & \\
\hline Total treatment duration (continuous) & 1 & $(0.99,1.00)$ & & \\
\hline
\end{tabular}

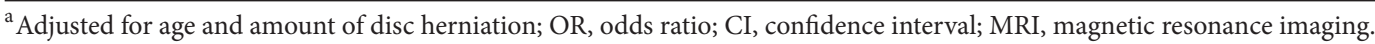

A recent trial on recovery of motor deficit which is widely considered to require surgical attention reported that differences between early surgery and prolonged conservative treatment at 1 year were nonsignificant [37]. Early surgery in lumbar IDH patients should be approached carefully and relevant information shared with patients in the decisionmaking process. Despite the fact that most participants were recommended for surgery in this study, not many were aware of spontaneous disc resorption.

Prospective large-scale studies further examining the disc resorption prediction process are warranted. Risk scores such as the Framingham Risk Score used to estimate 10-year cardiovascular risk may be similarly proposed in IDH through development of a prediction model to provide patients, physicians, and healthcare givers with valuable data necessary for informed decision-making in selecting IDH treatment.

\section{Conclusion}

In conclusion, the majority of patients who received conservative integrative Korean medicine treatment indicated a disc resorption volume of nearly $50 \%$ within a 1-year average, and the long-term course at 4.3 years was also favorable. IDH amount and type were identified as predictive factors associated with disc resorption and this information should be factored into prognosis and informed decision-making in treatment selection as most patients were unaware of the fact that disc resorption may occur spontaneously.

\section{Conflicts of Interest}

Jinho Lee and In-Hyuk Ha are employees of Jaseng Hospital of Korean Medicine and Jaseng Medical Foundation. JoonShik Shin is the chairman of Jaseng Hospital of Korean Medicine. Yoon Jae Lee is an employee of Jaseng Hospital of Korean Medicine. Joowon Kim, Me-riong Kim, Seon-Yeong Jeong, Young-jun Choi, Tae Kyung Yoon, Byung-heon Moon, Su-bin Yoo, and Jungsoo Hong are employees of Jaseng Medical Foundation.

\section{Authors' Contributions}

Jinho Lee and Joowon Kim contributed equally to this work as first authors.

\section{References}

[1] K. Konstantinou and K. M. Dunn, "Sciatica: review of epidemiological studies and prevalence estimates," Spine, vol. 33, no. 22, pp. 2464-2472, 2008. 
[2] M. W. van Tulder, B. W. Koes, and L. M. Bouter, "A cost-ofillness study of back pain in The Netherlands," Pain, vol. 62, no. 2, pp. 233-240, 1995.

[3] C. T. Merrill and A. Elixhauser, Hospitalization in the United States, 2002, Agency for Healthcare Research and Quality, Rockville, Md, USA, 2005.

[4] B. S. Gillum, E. Graves, and E. Wood, "National Hospital Discharge Survey," Vital and Health Statistics, vol. 13, no. 133, pp. $1-51,1998$.

[5] R. A. Deyo and S. K. Mirza, "Trends and variations in the use of spine surgery," Clinical Orthopaedics and Related Research, no. 443, pp. 139-146, 2006.

[6] R. A. Deyo and J. N. Weinstein, "Low back pain," The New England Journal of Medicine, vol. 344, no. 5, pp. 363-370, 2001.

[7] H. Weber, "Lumbar disc herniation: a controlled, prospective study with ten years of observation," Spine, vol. 8, no. 2, pp. 131140, 1983.

[8] W. C. Peul, W. B. Van Den Hout, R. Brand, R. T. W. M. Thomeer, and B. W. Koes, "Prolonged conservative care versus early surgery in patients with sciatica caused by lumbar disc herniation: two year results of a randomised controlled trial," $B M J$, vol. 336, no. 7657, pp. 1355-1358, 2008.

[9] S. Keskil, G. Ayberk, C. Evlıyaoğlu, T. Kizartici, E. Yücel, and H. Anbarci, "Spontaneous resolution of 'protruded' lumbar discs," Minimally Invasive Neurosurgery, vol. 47, no. 4, pp. 226-229, 2004.

[10] J.-Y. Seo, Y.-H. Roh, Y.-H. Kim, and K.-Y. Ha, “Three-dimensional analysis of volumetric changes in herniated discs of the lumbar spine: does spontaneous resorption of herniated discs always occur?" European Spine Journal, vol. 25, no. 5, pp. 13931402, 2016.

[11] G. L. Cvetanovich, A. R. Hsu, R. M. Frank, H. S. An, and G. B. Andersson, "Spontaneous resorption of a large cervical herniated nucleus pulposus," American journal of orthopedics (Belle Mead, N.J.), vol. 43, no. 7, pp. E140-E145, 2014.

[12] C.-C. Chiu, T.-Y. Chuang, K.-H. Chang, C.-H. Wu, P.-W. Lin, and W.-Y. Hsu, "The probability of spontaneous regression of lumbar herniated disc: a systematic review," Clinical Rehabilitation, vol. 29, no. 2, pp. 184-195, 2015.

[13] J. T. Oh, K. S. Park, S. S. Jung et al., "Surgical results and risk factors for recurrence of lumbar disc herniation," Korean Journal of Spine, vol. 9, no. 3, pp. 170-175, 2012.

[14] W. K. Erly, D. Munoz, and R. Beaton, "Can MRI signal characteristics of lumbar disk herniations predict disk regression?" Journal of Computer Assisted Tomography, vol. 30, no. 3, pp. 486-489, 2006.

[15] M. J. McGirt, S. Eustacchio, P. Varga et al., "A prospective cohort study of close interval computed tomography and magnetic resonance imaging after primary lumbar discectomy: factors associated with recurrent disc herniation and disc height loss," Spine, vol. 34, no. 19, pp. 2044-2051, 2009.

[16] C. Dora, M. R. Schmid, A. Elfering, M. Zanetti, J. Hodler, and N. Boos, "Lumbar disk herniation: do MR imaging findings predict recurrence after surgical diskectomy?" Radiology, vol. 235, no. 2, pp. 562-567, 2005.

[17] J. J. Park, J. Shin, Y. Choi et al., "Integrative package for low back pain with leg pain in Korea: a prospective cohort study," Complementary Therapies in Medicine, vol. 18, no. 2, pp. 78-86, 2010.

[18] J.-S. Shin, J. Lee, Y. J. Lee et al., "Long-term course of alternative and integrative therapy for lumbar disc herniation and risk factors for surgery: a prospective observational 5-year followup study," Spine, vol. 41, no. 16, pp. E955-E963, 2016.

[19] N. Robinson and J. Liu, "Oriental and traditional medicine-supporting the vision for integrated health," European Journal of Integrative Medicine, vol. 4, no. 4, pp. e363-e365, 2012.

[20] H. Komori, K. Shinomiya, O. Nakai, I. Yamaura, S. Takeda, and K. Furuya, "The natural history of herniated nucleus pulposus with radiculopathy," Spine, vol. 21, no. 2, pp. 225-229, 1996.

[21] C. W. A. Pfirrmann, A. Metzdorf, M. Zanetti, J. Hodler, and N. Boos, "Magnetic resonance classification of lumbar intervertebral disc degeneration," Spine, vol. 26, no. 17, pp. 1873-1878, 2001.

[22] H.-J. Chung, H.-S. Lee, J.-S. Shin et al., "Modulation of acute and chronic inflammatory processes by a traditional medicine preparation GCSB-5 both in vitro and in vivo animal models," Journal of Ethnopharmacology, vol. 130, no. 3, pp. 450-459, 2010.

[23] T.-H. Kim, S.-J. Yoon, W.-C. Lee et al., "Protective effect of GCSB-5, an herbal preparation, against peripheral nerve injury in rats," Journal of Ethnopharmacology, vol. 136, no. 2, pp. 297304, 2011.

[24] J.-K. Kim, S.-W. Park, J.-W. Kang et al., "Effect of GCSB-5, a herbal formulation, on monosodium iodoacetate-induced osteoarthritis in rats," Evidence-based Complementary and Alternative Medicine, vol. 2012, Article ID 730907, 11 pages, 2012.

[25] Y.-G. Park, C.-W. Ha, C.-D. Han et al., "A prospective, randomized, double-blind, multicenter comparative study on the safety and efficacy of Celecoxib and GCSB-5, dried extracts of six herbs, for the treatment of osteoarthritis of knee joint," Journal of Ethnopharmacology, vol. 149, no. 3, pp. 816-824, 2013.

[26] W. K. Kim, H.-J. Chung, Y. Pyee et al., "Effects of intra-articular SHINBARO treatment on monosodium iodoacetate-induced osteoarthritis in rats," Chinese Medicine, vol. 11, article 17, 2016.

[27] M. Doita, T. Kanatani, T. Ozaki, N. Matsui, M. Kurosaka, and S. Yoshiya, "Influence of macrophage infiltration of herniated disc tissue on the production of matrix metalloproteinases leading to disc resorption," Spine, vol. 26, no. 14, pp. 1522-1527, 2001.

[28] T. Henmi, H. Endo, S. Nakano, and K. Sairyo, "Natural history of extruded lumbar intervertebral disc herniation," The Journal of Medical Investigation, vol. 49, no. 1/2, pp. 40-43, 2002.

[29] S.-H. Ahn, M.-W. Ahn, and W.-M. Byun, "Effect of the transligamentous extension of lumbar disc herniations on their regression and the clinical outcome of sciatica," Spine, vol. 25, no. 4, pp. 475-480, 2000.

[30] J.-Y. Maigne, B. Rime, and B. Deligne, "Computed tomographic follow-up study of forty-eight cases of nonoperatively treated lumbar intervertebral disc herniation," Spine, vol. 17, no. 9, pp. 1071-1074, 1992.

[31] H. Komori, A. Okawa, H. Haro, T. Muneta, H. Yamamoto, and K. Shinomiya, "Contrast-enhanced magnetic resonance imaging in conservative management of lumbar disc herniation," Spine, vol. 23, no. 1, pp. 67-73, 1998.

[32] Z. Shan, S. Fan, Q. Xie et al., "Spontaneous resorption of lumbar disc herniation is less likely when modic changes are present," Spine, vol. 39, no. 9, pp. 736-744, 2014.

[33] M. Iwabuchi, K. Murakami, F. Ara, K. Otani, and S.-I. Kikuchi, "The predictive factors for the resorption of a lumbar disc herniation on plain MRI," Fukushima Journal of Medical Science, vol. 56, no. 2, pp. 91-97, 2010.

[34] G. B. Andersson, M. D. Brown, J. Dvorak et al., "Consensus summary on the diagnosis and treatment of lumbar disc herniation," Spine, vol. 21, no. 24, pp. 75S-78S, 1996. 
[35] P. A. J. Luijsterburg, A. P. Verhagen, S. Braak, C. J. J. Avezaat, and B. W. Koes, "Do neurosurgeons subscribe to the guideline lumbosacral radicular syndrome?" Clinical Neurology and Neurosurgery, vol. 106, no. 4, pp. 313-317, 2004.

[36] J.-P. Vader, F. Porchet, T. Larequi-Lauber, R. W. Dubois, and B. Burnand, "Appropriateness of surgery for sciatica: reliability of guidelines from expert panels," Spine, vol. 25, no. 14, pp. 1831$1836,2000$.

[37] G. M. Overdevest, C. L. A. M. Vleggeert-Lankamp, W. C. H. Jacobs, R. Brand, B. W. Koes, and W. C. Peul, "Recovery of motor deficit accompanying sciatica-subgroup analysis of a randomized controlled trial," Spine Journal, vol. 14, no. 9, pp. 18171824, 2014. 


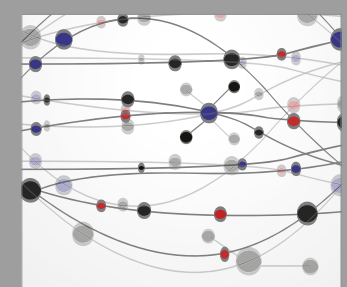

The Scientific World Journal
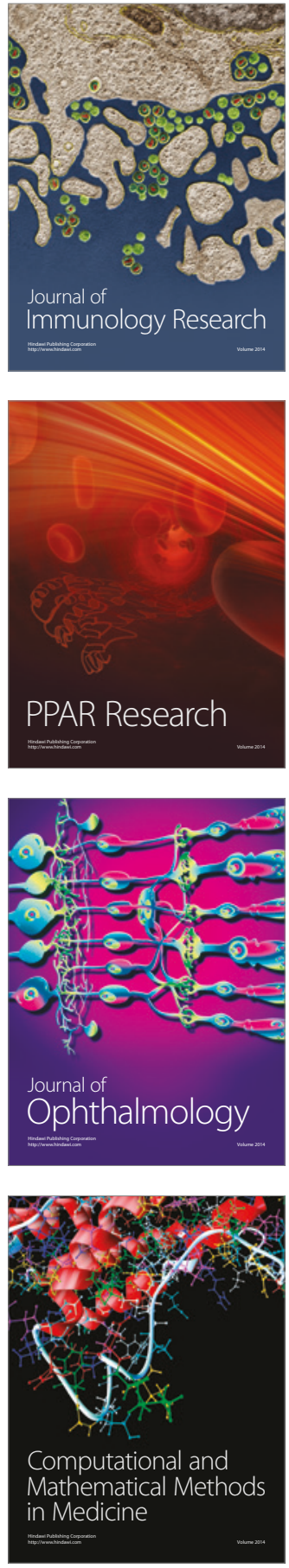

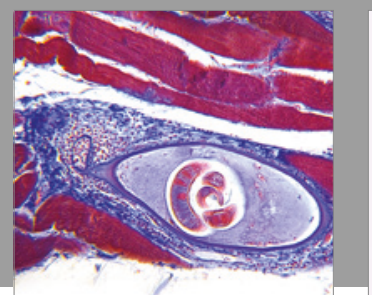

Gastroenterology Research and Practice
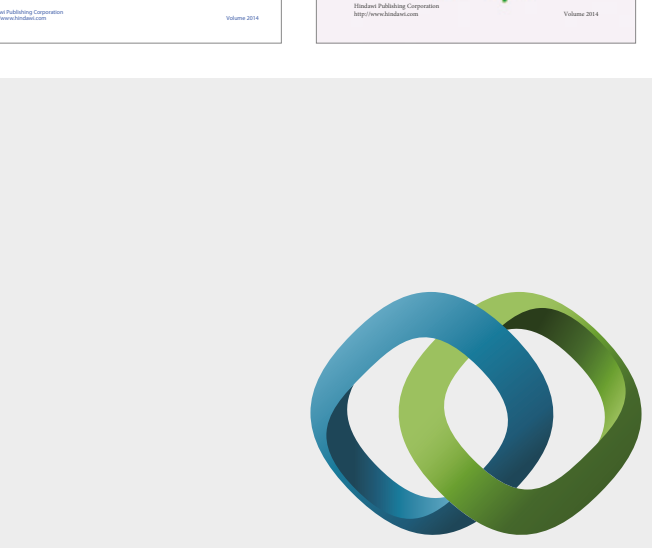

\section{Hindawi}

Submit your manuscripts at

https://www.hindawi.com
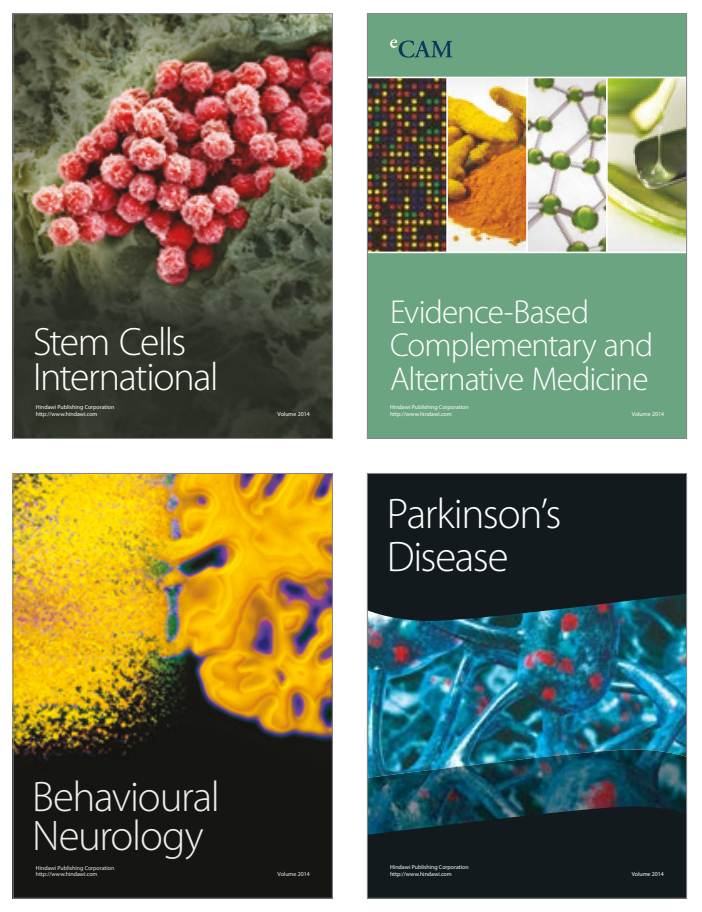
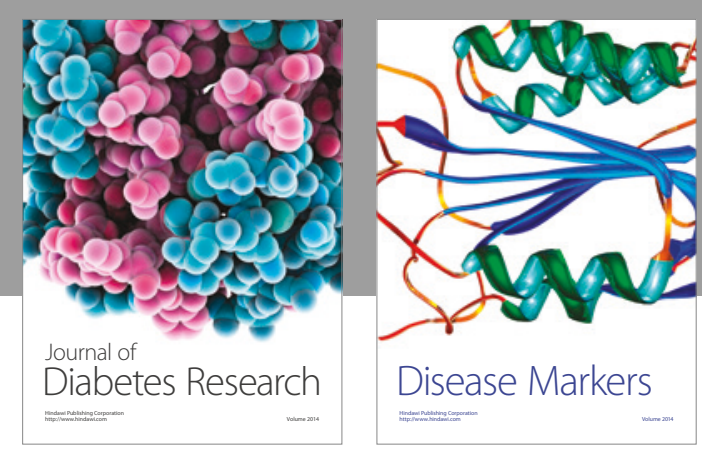

Disease Markers
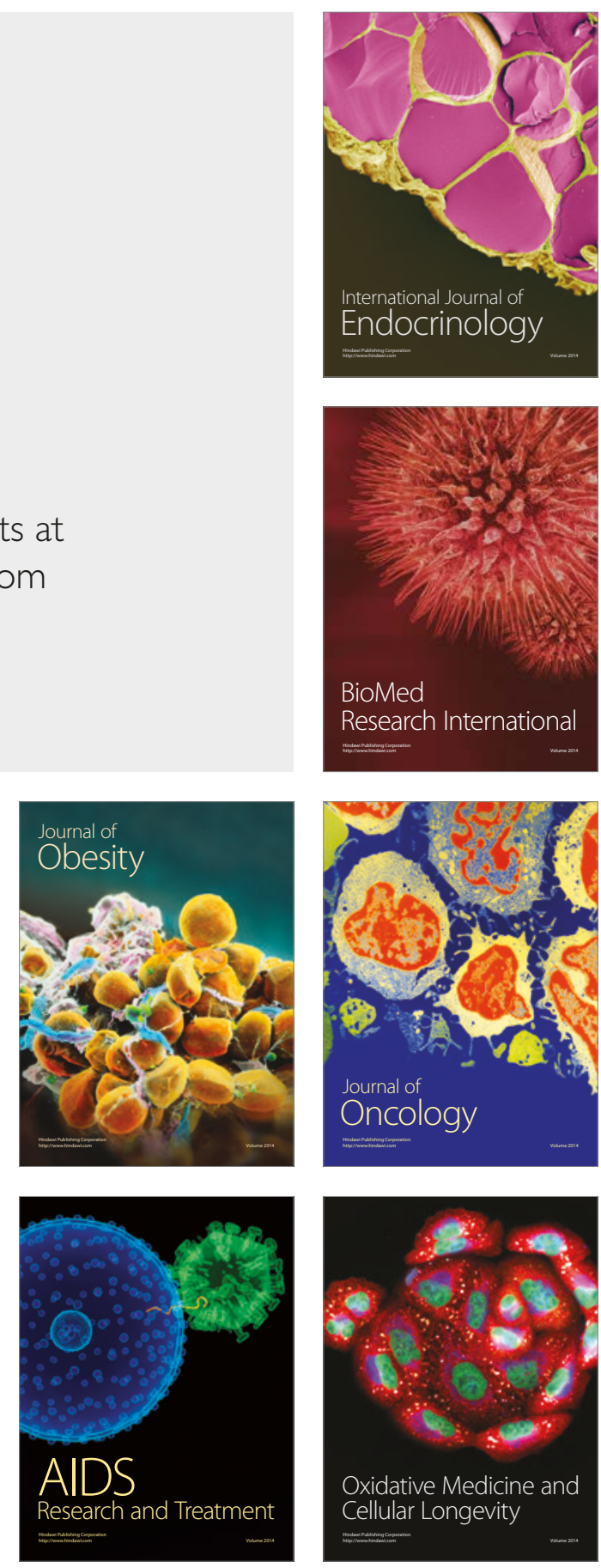